\title{
TaAAP6-3B, a regulator of grain protein content selected during wheat improvement
}

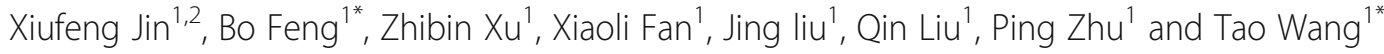

\begin{abstract}
Background: The content of grain protein (GPC) in cereals is an important part of total protein in human food. Exploring and utilizing new GPC genes is one of the most effective approaches for wheat quality breeding.

Results: Three homoeologues of TaAAP6(-3A, 3B, 3D)were cloned by homology cloning from OsAAP6.Temporal and spatial expression analysis showed that TaAAP6homoeologues were preferentially expressed in developing grains, and TaAAP6-3B may play a major role in regulating GPC in wheat. Association analysis indicated thatTaAAP6-3B-I is significantly correlated with higher GPC than that of TaAAP6-3B-I/ for 115 wheat lines in all five environments. TaAAP6-3B-I, the favored allele of TaAAP6-3B, was preferentially expressed in preliminary developing grain stage. Two functional markers were developed to discriminate 197F 2 populations and the result showed that TaAAP6-3B-I (high-protein content) was completely dominant. Two cis-regulatory elements appear to be associated with high GPC were found in the 5'UTR of TaAAP6-3B-I.The change of the TaAAP6-3B locus types indicated that the gene was subjected to selection pressures during long process of artificial selection.
\end{abstract}

Conclusions: TaAAP6-3B is a regulator of GPC and its favored allele TaAAP6-3B-/ exhibits an obvious potential application in wheat high-GPC breeding.

Keywords: GPC, Triticumaestivum L., TaAAP6-3B, Selection pressure

\section{Background}

Enhancing nutritional quality of cereals is a means of improving human nutrition and health [1]. Nearly one-third of the world population currently suffers from protein malnutrition, and some diseases are caused by poor quality protein and lack of vitamins and other micro-nutrients [2]. The content of grain protein (GPC) in cereals is an important part of total protein in human food. Bread wheat (Triticum aestivum L.) is one of the most important food crops worldwide, accounting for $\sim 20 \%$ of all calories and approximately $10 \%$ of production transforming into protein were consumed [3]. Moreover, the demand for high-quality wheat as a source of protein is expected to increase dramatically in the near future. Cloning GPC-related genes, exploring the favored

\footnotetext{
*Correspondence: fengb2600@163.com; wangtao@cib.ac.cn

${ }^{1}$ Chengdu Institute of Biology, Chinese Academy of Sciences, Chengdu, China

Full list of author information is available at the end of the article
}

alleles and developing functional markers could be used in Molecular Module-Based Designer Breeding Systems for wheat.

Common wheat is a hexaploid specie (AABBDD) with a large genome size $(17.9 \mathrm{~Gb})$ and abundant repeat sequences (> 80\%) [4]. Comparative genomics proved the existence of chromosomes colinearity between wheat and rice. Recently, a few wheat genes were successfully cloned by comparative genomics, such as TaGW2 [5], TaGS5 [6], TaSus1 [7], TaSus2 [8] et al. Therefore, in combination with completion of a draft for wheat genome sequence, homology-based cloning has become an efficient way to isolate genes in wheat.

GPC is a typical quantitative trait controlled by a complex genetic regulation and influenced by environmental factors and management practices. So far, numerous quantitative trait loci (QTL) associated with GPC have been detected in a number of environments and populations [9-12]. However it is difficult to directly isolate GPC-related genes by map-based cloning strategies due 
to its huge and complex genome except $G p c-B 1$ on $6 \mathrm{BS}$ [1], whose identification is significantly meaningful for nutritional quality in cereal crops. Previous studies revealed a putative amino acid transporter OsAAP6, which functions as a positive regulator of GPC in rice [3]. As yet, no information is available on the expression of respective homologues of the candidate gene for high contents of grain protein in common wheat. Therefore, our objectives were to clone homologues of TaAAP6, and analyze the temporal and spatial expression of them to confirm candidate gene. Association analyzes of GPC haplotypes alleles for 115 wheat cultivars in all five environments. Evaluate potential cis-elements associated with GPC diversity. And then, in order to understand the selection intensity of candidate gene block in manual selective breeding, the high-density wheat $90 \mathrm{~K}$ Illuminai Select SNP array [13] were selected to find polymorphism of the locus block.

\section{Results}

TaAAP6-3B、3D show high identity to OsAAP6

Based on the conserved sequences of OsAAP6 (NCBI Accession number:KM213630), TaAAP6 genomic and CDS were blast from wheat genome in Chinese Spring, and analytical scorings were likely to located on 3A、3B、3D chromosome. Their genomic sequence lengths were 4379, 3841 and $3898 \mathrm{bp}$, encoding putative 492, 467 and 467 amino acids respectively. Full-length CDNA of the genes were amplified by 5 -UTR and 3'-UTR genome-specific primers, $A F c, B F c, D F c$ (Additional file 1: Table S2). Compare to four exons in OsAAP6, TaAAP6-3A and TaAAP6-3B consisted of four exons, and TaAAP6-3D consisted of five exons (Fig. 1). All of them contain a large first intron, which is similar to that of OsAAP6.

The cluster analysis of TaAAP6 patterns for the three genes showed that the similarities between them are up to $75.90 \%$. Based on the amino acids sequences and domains, pair wise comparison result showed that TaAAP6-3B - TaAAP6-3D shared a higher identity (97. 86\%) than that of TaAAP6-3A - TaAAP6-3D (57.40\%) and TaAAP6-3A - TaAAP6-3B (58.42\%). Moreover, TaAAP6-3A contained five insertions, and there are only six different amino acid residues between TaAAP6-3B and TaAAP6-3D. Sequence alignment result shows that TaAAP6-3B and TaAAP6-3D were more similar to OsAAP6 thanTaAAP6-3A (Additional file 2: Figure S1).

\section{Phylogenetic analyses}

Amino acid transporters/permeases are key regulators of plant metabolism, growth and development [14]. A phylogenetic analysis was performed by generating a neighbor-joining phylogenetic tree (Fig. 2), consist of one member ( $Z m A A P 4)$ in maize, eight members (AtAAP1-8) in Arabidopsis,18 members (OsAAP1-18) in rice (http://aramemnon.botanik.uni-koeln.de/). Five clusters were formed according to differences in amino acid sequences of AAPs, both TaAAP6 and OsAAP6 are classed as a subgroup, which means TaAAP6 may be the ortholog of OsAAP6, and TaAAP6-3B was closer to TaAAP6-3D than to TaAAP6-3A (Fig. 2). Previously, the $T a A A P$ family have been mapped to wheat chromosome and it has been named as TaAAP8 [15].

\section{TaAAP6-3B was preferentially expressed in developing grains}

In order to investigate the temporal and spatial expression patterns of the TaAAP6 homoeologues in different tissues and organs, specific primers $q A, q B$ and $q D$ (Additional file 1: Table S2) were used for quantitative real-time PCR (qRT-PCR). The three homoeologues were ubiquitously expressed with similar patterns in various tissues, but showed higher expression in developing grains than in established roots and seedlings (Fig. 3). The most abundant expression was at 7 days after flowering (DAF) in endosperm, then gradually declined until $21 \mathrm{DAF}$, and increased at 28 DAF. This result showed a similar "highlow-high" dynamic trend to the protein content change during grain filling stage [16]. Although three homologous genes showed similar expression patterns, they had significantly different expression abundances. Compared to TaAAP6-3A and TaAAP6-3D, TaAAP6$3 B$ was expressed at a much higher level, one and half timesto that of TaAAP6-3A and three times to that of TaAAP6-3D (Fig. 3).

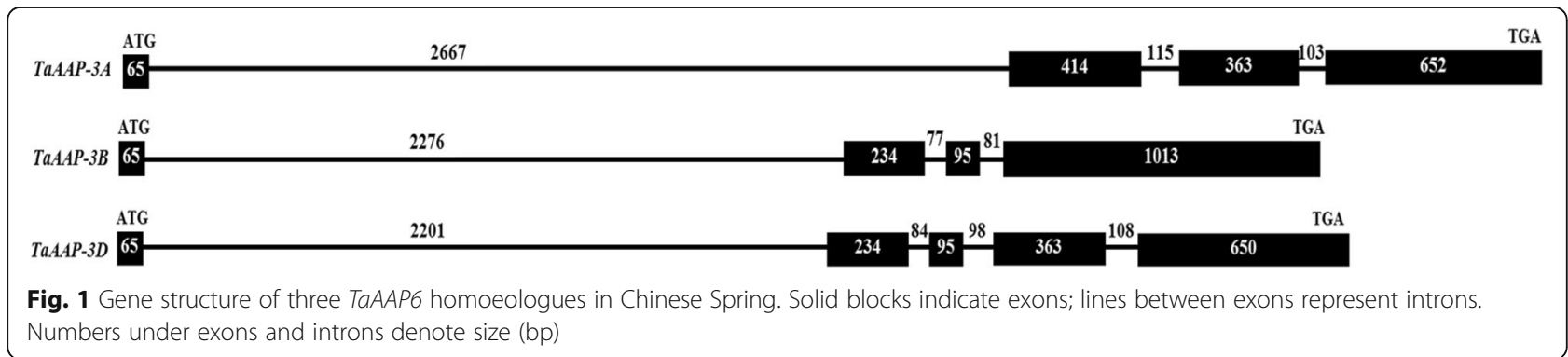




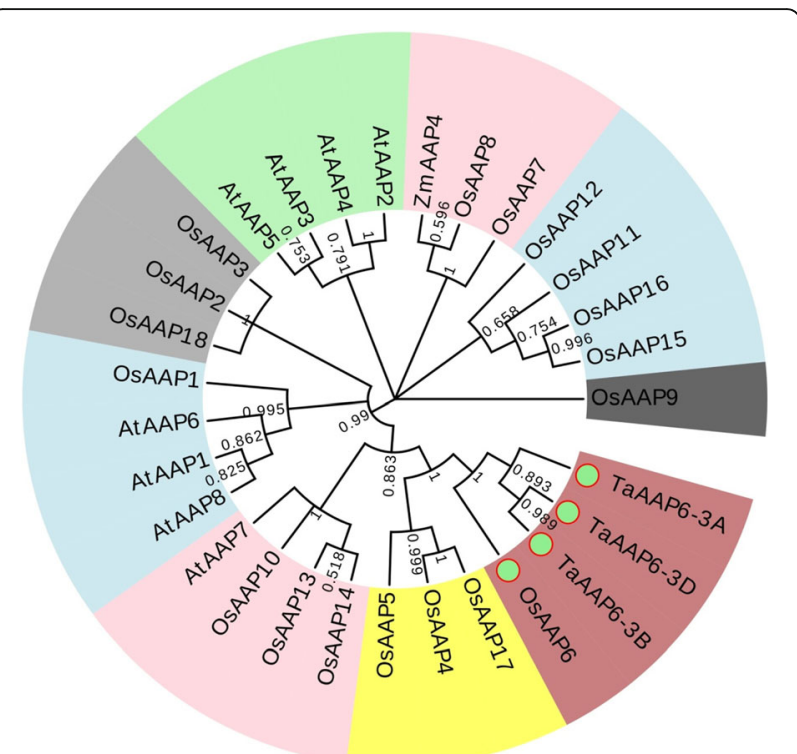

Fig. 2 Phylogenetic analyses of the AAPs. At (Arabidopsis thaliana), Os (Oryza sativa) and Zm (Zea mays). The genes are as follows: AtAPP1(Gene ID: At1g58360), AtAPP2 (Gene ID: At5g09220), AtAPP3(Gene ID: At1g77380), AtAPP4(Gene ID: At5g63850), AtAPP5(Gene ID: At1g44100), AtAPP6(Gene ID: At5g49630), AtAPP7(Gene ID: At5g23810), AtAPP8(Gene ID: At1g10010), OsAPP1(Gene ID: LOC_Os07g04180), OsAPP2(Gene ID: LOC_Os06g12330), OsAPP3(Gene ID: LOC_Os06g36180), OsAPP4(Gene ID: LOC_Os12g09300), OsAPP5(Gene ID: LOC_Os01g65660), OsAPP6(Gene ID: LOC_Os01g65670), OsAPP7(Gene ID: LOC_Os05g34980), OsAPP8(Gene ID: LOC_Os01g66010), OsAPP9 (Gene ID: LOC_Os02g01210), OsAPP10(Gene ID: LOC_Os02g49060), OsAPP11 (Gene ID: LOC_Os11 g09020), OsAPP12(Gene ID: LOC_Os12g09320), OsAPP13(Gene ID: LOC_Os04g39489), OsAPP14(Gene ID: LOC_Os04g56470), OsAPP15(Gene ID: LOC_Os12g08130), OsAPP16(Gene ID: LOC_Os12g08090), OsAPP17(Gene ID: LOC_Os06g12350), OsAPP18(Gene ID: LOC_Os06g36210), ZmAPP4(Gene ID: GRMZM2G110195). The phylogenetic tree is produced by online software (http://www.evolgenius.info/evolview/ \#login) based on the comparison of amino acid sequences

\section{Allelic variations of TaAAP6-3B}

To detect sequence variations of TaAAP6-3B, three pairs of primers $B F 1, B F 2$ and $B F 3$ were designed to amplify the genome-specific fragments, and full-length sequence of TaAAP6-3B gene was assembled. Based on the nucleotide polymorphisms identified among the 115 Chinese wheat accessions (Additional file 3: Table S1), the sequences were divided into two haplotypes, which were named TaAAP6-3B-I and TaAAP6-3B-II. 17 synonymous mutation sites were identified in genomic sequence between them, and the coding regions contained 4 synonymous mutation sites (Fig. 4). Meanwhile, ten sites were detected in the promoter region, and a nucleotide deletion was found in TaAAP6-3B-II.
Two Cis-acting elements associated with GPC were found in TaAAP6-3B-I

Previous studies have shown that, Cis-regulatory elements (CREs) can impact gene expression levels, but also developmental timing and tissue specificity of expression [17]. In rice, OsAAP6 expression was regulated by three CREs, including Sulphur-responsive element, copper-response element and inr-element in high GPC population [3]. In TaAAP6-3B, promoter region $(\sim 1$. $8 \mathrm{~kb})$ was amplified by specific primers $B F 1$ and $B F 2$ (Additional file 1: Table S2). The result shows that six CREs in upstream sequence were identified in TaAAP6$3 B-I$ and not detected inTaAAP6-3B-II(Additional file 4: Table S4). Two CREs target for transcriptional activators and regulators may be associated with high GPC for them. One (-753 bp) is a Sulfur-responsive element [18, 19], another $(\sim 1009 \mathrm{bp})$ is a SEF4 motif which interacts with a soybean storage protein enhancer [20]. Taken together, our results imply that the two common variations in the two potential CREs of the TaAAP6-3B 5'-UTR appear to be associated with GPC diversity in the populations.

TaAAP6-3B- I shows a much higher expressing level In order to investigate the difference expression patterns of the two haplotypes, two primers Actin1, $q B$ (Additional file 5: Figure S2) were used to detect the expression patterns through qRT-PCR in modern cultivars. TaAAP6-3B$I$ and TaAAP6-3B-II was expressed with similar pattern in six tissues. However, they had significantly different expression abundances. Compared to TaAAP6-3B-II, TaAAP6-3B-I was expressed at a much higher level in every stage,especially in the endosperm at $7 \mathrm{DAF}$, the expression excelled two times (Additional file 5: Figure S2).

\section{TaAAP6-3B-I associates with high GPC in natural and genetic populations}

The content of grain protein (GPC) was measured in 115 hexaploid wheat lines. Compared to 51 modern cultivars, the 64 landraces exhibited a significantly higher GPC in all five environments $(P<0.001)$ (Fig. 5a) and the phenotypic differences between the two lines were 20.31, 23.33, 21.66, 9.04, and 9.96(\%), respectively (Additional file 6: Table S3).

After genotyping, association analysis between phenotypes and genotypes was performed. The results show that the GPC of TaAAP6-3B-I issignificantly higher thanthat of TaAAP6-3B-II $(P<0.001)$ (Fig. 5b), and differences between the two haplotypes were 17.94, 20.85, 19.58, 9.29 and 8.53 (\%) in all five environments, respectively (Additional file 6: Table S3). Furthermore, in landraces, the significant differences between 53 TaAAP6-3B-I lines and 11 TaAAP6-3B-II lines were detected in four environments (Fig. $5 \mathrm{c}$ ). The mean differences between the two alleles were 7.50, 9.55, 13.77, 


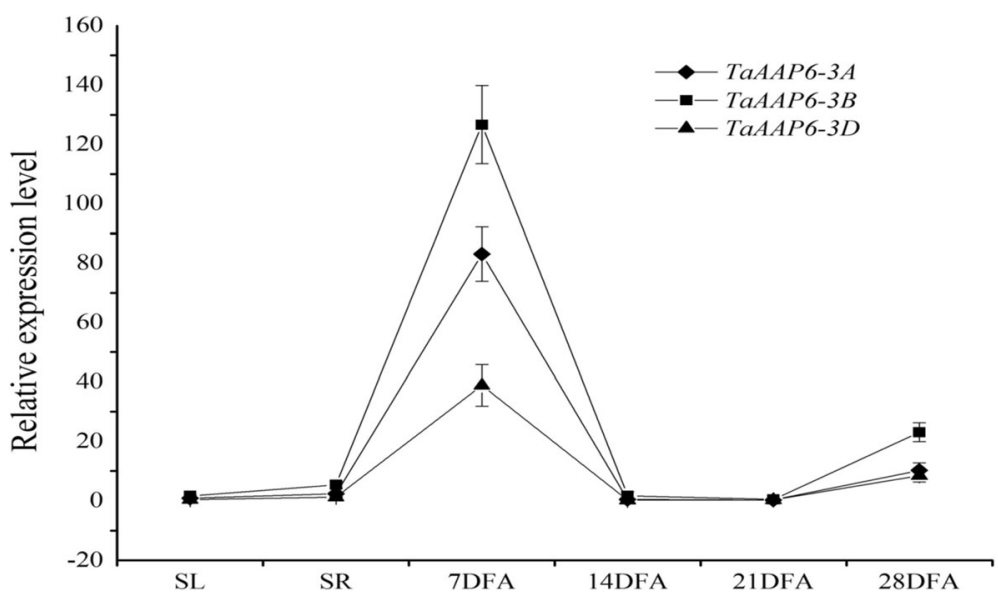

Fig. 3 The comparative expression pattern of TaAAP6 homoeologues. SL, seedling leaf; SR, seedling root; various stages of grain development, including 7DAF, 14DAF, 21DAF, 28DAF (days after flowering). Error bars denote \pm SD

and 9.37 (\%), respectively (Additional file 6: Table S3). For modern cultivars, the two haplotypes detected in three environments have significant differences (Fig. 5d). The mean differences were 9.66, 10.69 and 4.87(\%), respectively (Additional file 6: Table S3).

To further distinguish the twohaplotypes of TaAAP6$3 B$, two pairs of complementary primers (Additional file 1: Table S2) were designed. The PCR product of TaAAP6-3B-I amplified by the genomic-specific primer pairs $B I$ was $288 \mathrm{bp}$, whereas that of TaAAP6-3B-II has not emerged. And for TaAAP6-3B-II, $539 \mathrm{bp}$ band could be amplified with primer pairs $B I I$, whereas that of TaAAP6-3B-I has not emerged (Fig. 6a).

The two markers were also used for detecting genetic populations (Fig. $6 \mathrm{~b} 1, \mathrm{~b} 2$ ). Analysis of a $\mathrm{F}_{2}$ population containing 197 individuals derived by ZM5453 (TaAAP6$3 B-I) \times$ ZhongKemai138 (TaAAP6-3B-II) showed that GPC in heterozygous plants was significantly lower than that in TaAAP6-3B-I homozygotes but higher than that in TaAAP6-3B-II homozygotes (Additional file 7: Table S5), which indicates that the high-protein content allele TaAAP6-3B-I was completely dominant.

\section{Changing of the locus polymorphism during wheat improvement}

Domestication and modern plant breeding have previously narrowed the genetic base of bread wheat. The detection of loci under selection during crop improvement can contribute to more targeted breeding efforts and the opportunity to improve genomic selection models [21]. In order to detect the variation of the locus polymorphism during artificial selection, 40 high-density SNPs around the TaAAP6-3B locus on $3 \mathrm{~B}$ chromosome was used for genotyping in 115 wheat lines, and four polymorphic SNPs were found (Fig. 7).

For wsnp_Ku_c38911_47455924, three variations AA, AG, GG were found. And two variations GG, AG; GG, AG and TC, CC were detected at BS00048355_51; RAC875_c39926_313 andBS00090869_51, respectively. These four SNPs constitute 24 types of the locus polymorphism in theory; nevertheless only 14 types were found in our study (Additional file 6: Table S3). With all 14 types among landraces, type 11 constituted 35.3\%; and type $6(23.5 \%)$; type 8 (11.8\%); type 3 (7.4\%); type 2 (5.9\%) and the remaining under 5\%, respectively. Of all 9 typesin cultivars, type 1 constituted $37.5 \%$, and type 10 (20.8\%); type $9(18.8 \%)$; type $5(10.4 \%)$; type $2(6.3 \%)$ and the remaining under $5 \%$, respectively(Fig. 8).

Meanwhile, five locus types $(4,5,8,11,14)$ in landraces have been selected off, of which type 8 and 11 account for $70.6 \%$ lines among SubI. Interestingly, three types (1, 9 and 10) account for only $8.8 \%$ lines in landraces, but account for $64.6 \%$ lines in SubII for cultivars. The above results show that, after artificial selection, the SubI (high GPC populations) were gradually replaced by SubII (low GPC populations) (Fig. 8).

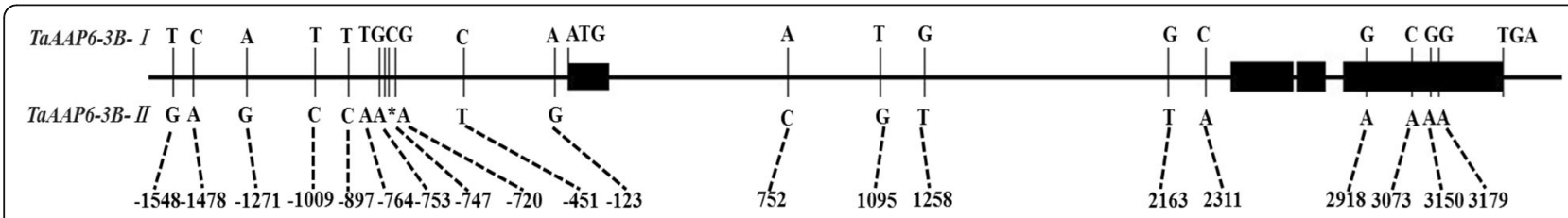

Fig. 4 Gene structure and natural variation of the two TaAAP6-3B haplotypes 

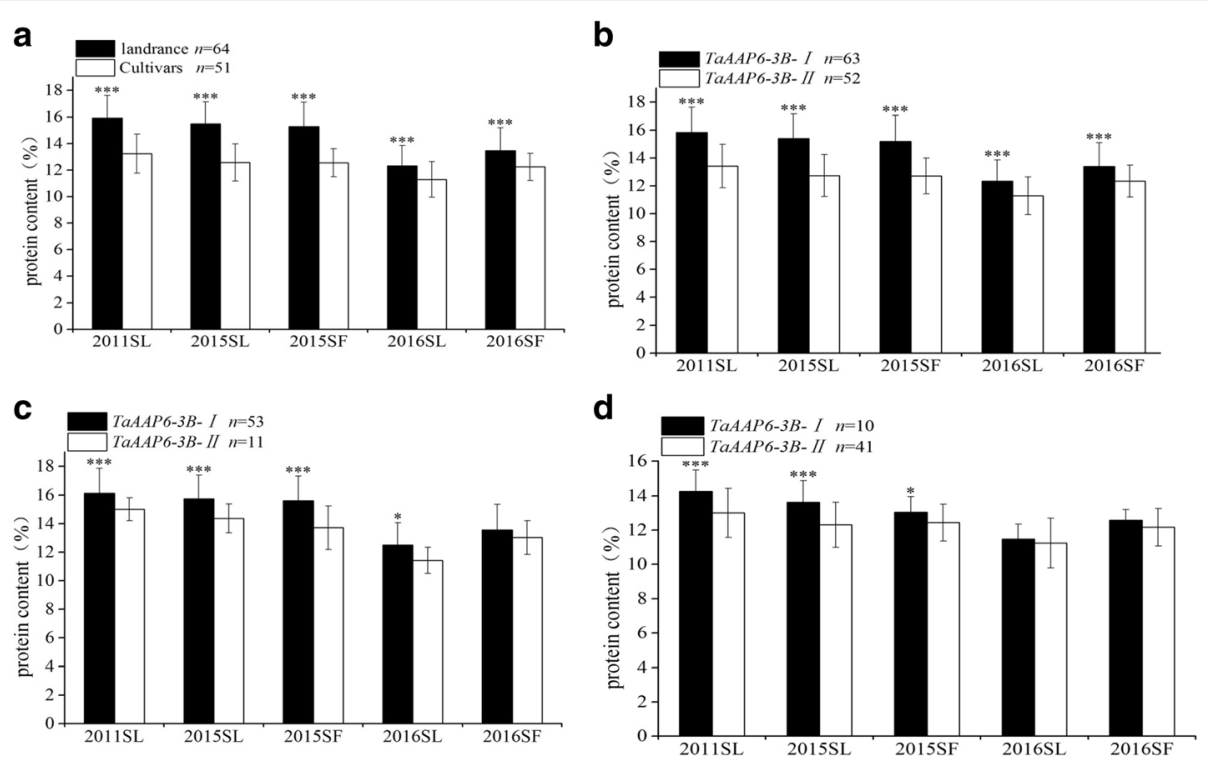

Fig. 5 Correlation analyses of grain protein contents (GPC) in 115 lines. a The GPC between landraces and modern cultivars; $\mathbf{b}$ The GPC between TaAAP6-3B-I and TaAAP6-3B-II; c The GPC between two haplotypes in landraces; $\mathbf{d}$ The GPC between two haplotypes in modern cultivars. $\mathrm{n}$, is the number of accessions. Significant differences at ${ }^{*} P=0.05,{ }^{* *} P=0.01$ and ${ }^{* * *} P=0.001$, respectively. Error bars denote \pm SD

\section{Discussion}

\section{TaAAP6-3B regulates GPC similar to OsAAP6}

Wheat GPC is a multigenic-controlled complex trait, and the demand for high-quality cereals as a source of protein has become increased [15]. So far, only NAM1 gene has been reported to enhance the nutritional value of crops, which was associated with increased GPC in bread wheat [1]. Efforts to identify more genes affected GPC and direct selection of the alleles with positive effects is necessary.
Physiological data further suggest that amino acid permeases are key regulators in plant metabolism and that their activities affect growth and development $[16,17]$. In Arabidopsis, AtAAP1 and AtAAP5 are important to uptake of neutral and acidic amino acids [18]; AtAAP2 to sinks is decreased leading to reduce total nitrogen and protein levels in seeds [22]. AtAAP8 mediates amino acid uptake into the endosperm at the early embryo stage [20]. In rice, OsAAP8 and OsAAP15 might participate in the uptake and long-distance transport of amino

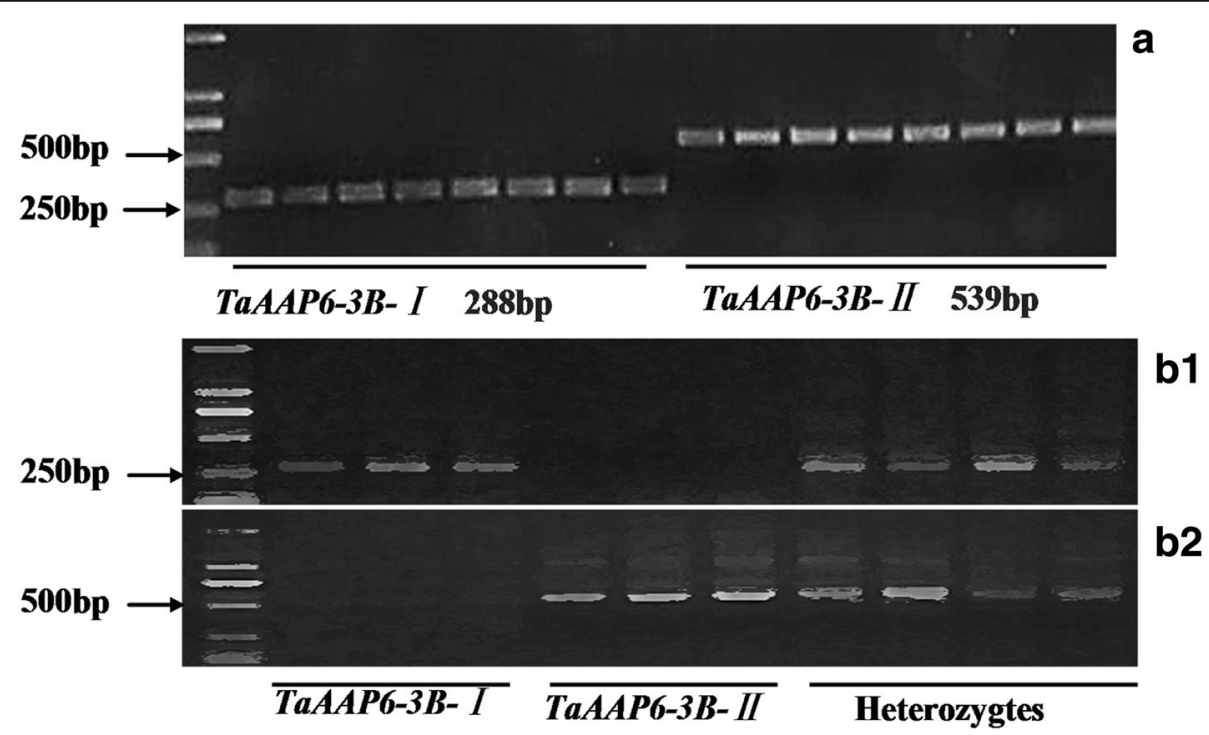

Fig. 6 Two markers developed for identify two haplotypes of TaAAP6-3B. (a) PCR products of TaAAP6-3B in natural populations; (b1), (b2) PCR products of TaAAP6-3B in $\mathrm{F}_{2}$ populations 


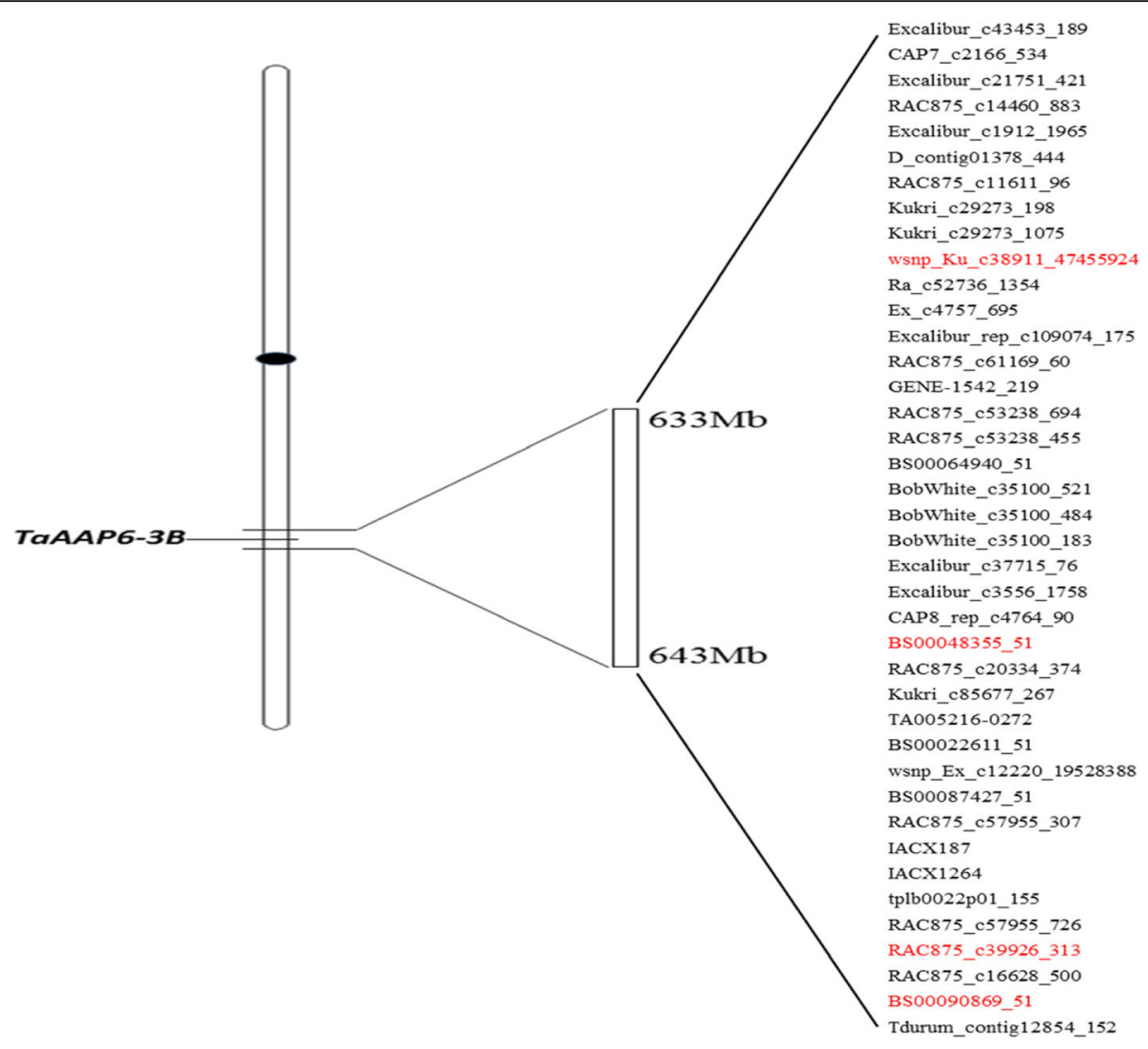

Fig. 740 SNPs in the TaAAP6-3B locus on 3B chromosome. The reds are the polymorphic SNPs in 115 lines

acid [23], OsAAP6 has pleiotropic effects on grain storage materials.

Our study showed that TaAAP6 is a member of AAP family genes (Fig. 2), and very closer to OsAAP6. QRT-PCR results show that TaAAP6-3B has the highest express level, which suggests it may be the important homologue. Allelic variations in 115 wheat lines contained two haplotypes TaAAP6-
3B-I and TaAAP6-3B-II. The expression pattern at stages of seed development showed TaAAP6-3B-I in developing seeds were significantly positively associated with higher GPC than TaAAP6-3B-II (Additional file 5: Figure S2). Association analysis showed that TaAAP6-3B-I was a favored allele associated with higher GPC both in natural and genetic populations.

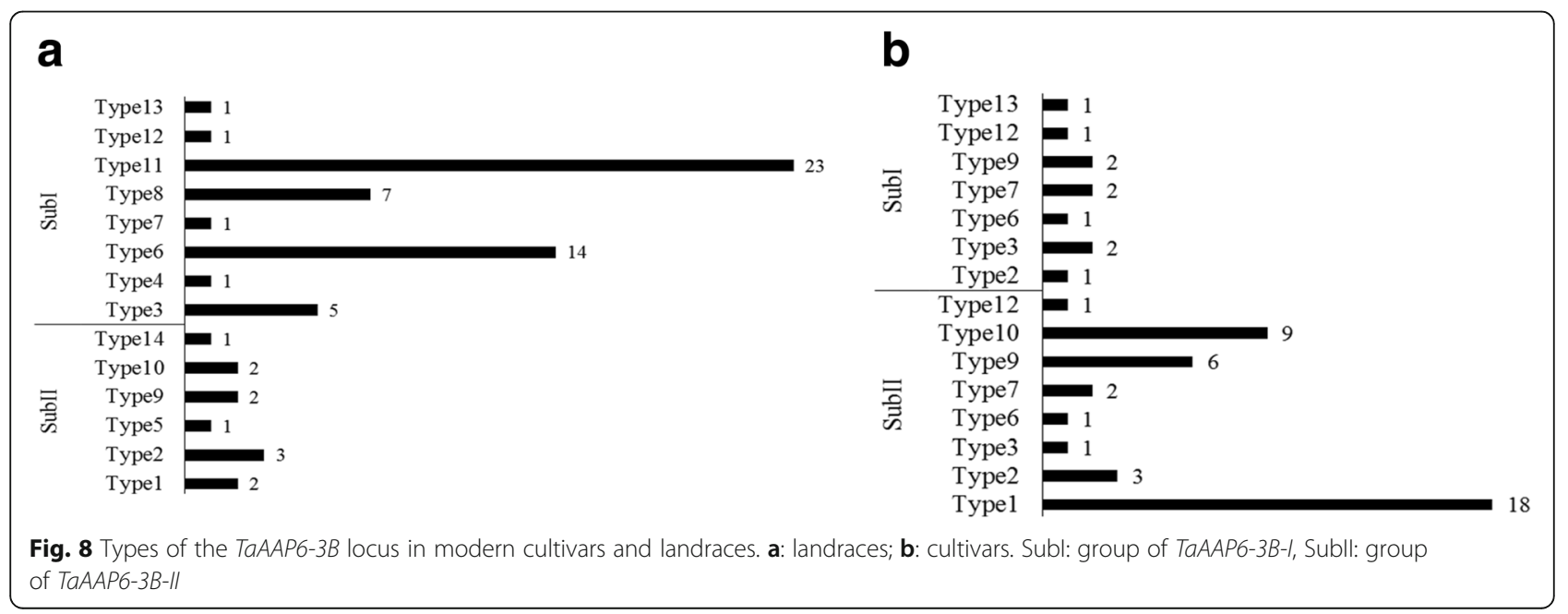


Cis-Regulatory elements (CREs) control the expression of an unmodified coding sequence during artificial selection in crops. In-depth understanding of gene regulatory networks and genome editing to find and alter CREs at the single nucleotide level in plant genomes may provide a promising engineering strategy for future crop improvement [24]. In rice, three CREs, Sulphur-responsive element, copper-response element and inr-element are key factors to be associated with GPC diversity [3]. Our results showed that two CREs may play important roles in controlling wheat GPC by regulating the TaAAP6-3B expression. One is sulphur-responsive element, the binding sequence of auxin response factor, which targets for transcriptional activators and involved in a broad range of responses $[18,19]$. And another is SEF4 motif, which was found to increase in soybean embryos during the time of $\beta$-conglycinin synthesis [20].

TaAAP6-3B-I has subjected to strong selection pressure in wheat improvement

Wheat (Triticum aestivum L.) is one of the founder crops that likely drove the Neolithic transition to sedentary agrarian societies in the Fertile Crescent more than 10,000 years ago [25]. During the past centuries, so many traditional landraces were continually replaced by modern cultivars to adapt to specific conditions of human requirements [26]. The genetic variation was subsequently reduced by selection especially for genes controlling agronomic traits, such as TKW and GPC.

Previous results suggest a negative relationship between TKW and GPC [27, 28]. Grain yield, the primary breeding objective, has been substantially increased during selection. TKW, a major component of yield, has translated into continuous genetic gains [29]. In this study, we compared the TKW of TaAAP6-3B-I and TaAAP6-3B-II in modern cultivars and landraces at all five environments (Additional file 1: Table S2). The result also revealed the negatively relationship between TKW and GPC (Figs. 5 and 9). This means in the long-term selection of breeding, the GPC gradually decreased, while the TKW gradually increased. It was also confirmed that both traits are the products of complex interdependencies between plant developmental traits and yield components. In our $197 \mathrm{~F}_{2}$ genetic populations, the number of TaAAP6-3B-I homozygotes is two times of TaAAP6-3B-II homozygotes. This phenomenon could be explained due to the physical interval associated with segregation distortion loci [30, 31]. Although the ration of TaAAP6-3B-I is much higher in a segregation population (41.62\%) (Additional file 7: Table $\mathrm{S} 5$ ), its ration in modern varieties is lower (19.61\%) (Fig. $5 d)$. It suggests that TaAAP6-3B-I haplotype was selected off during artificial selection. The similar results can be found in changing of TaAAP6-3B haplotype block types. In landraces, TaAAP6-3B-I associate with high GPC and low TKW is dominant(82. $81 \%)$. In modern varieties, TaAAP6-3B-II associate with low GPC and high TKW is dominant (82.00\%). As we know, the main objective of breeding is focus on yield, and TKW is under a positive selection. Because of the negative relationship of $\mathrm{GPC}$ and TKW, GPC may under a negative selection. As a result, all these findings indicate that TaAAP6-3B gene
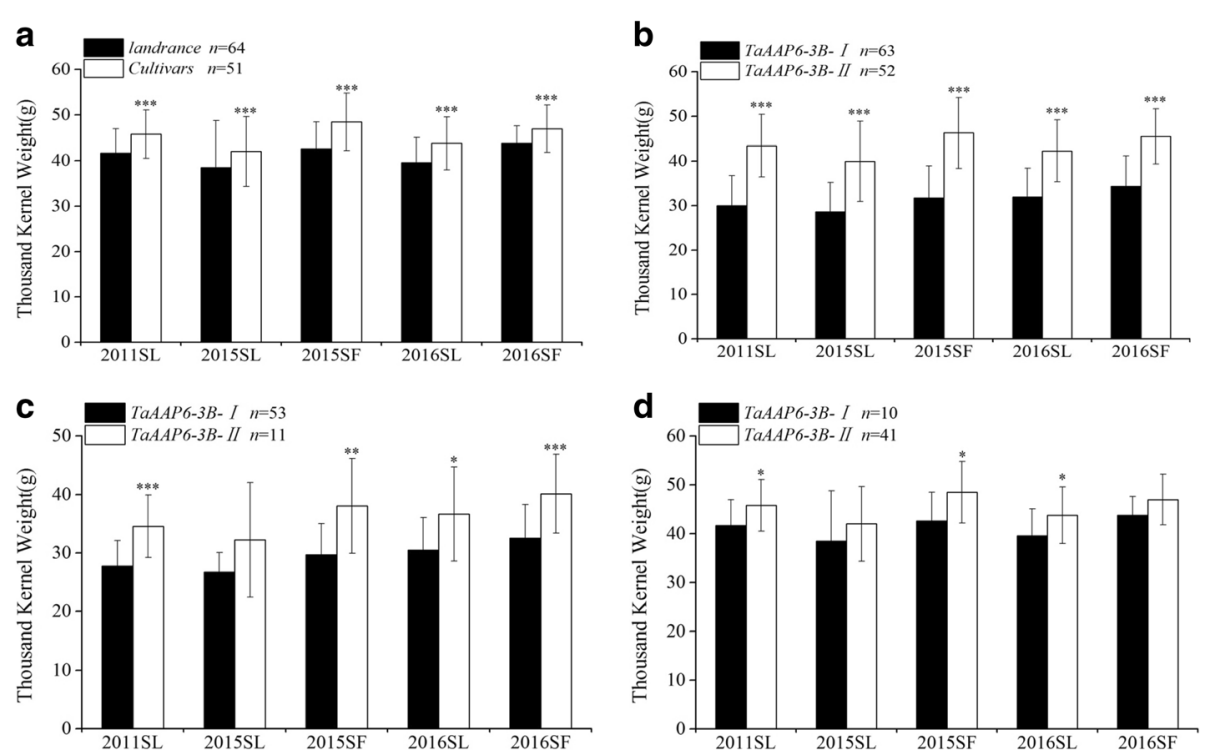

Fig. 9 Correlation analyses of thousand-kernel weight (TKW) in 115 lines. a The TKW between landraces and modern cultivars; $\mathbf{b}$ TKW between phenotypic of TaAAP6-3B-I and TaAAP6-3B-II; c The TKW between two haplotypes in landraces; $\mathbf{d}$ The TKW between two haplotypes in modern cultivars. $n$, is the number of accessions. Significant differences at ${ }^{*} P=0.05,{ }^{*} P=0.01$ and ${ }^{* *} P=0.001$, respectively. Error bars denote \pm sd 
has subjected to strong selection pressure in wheat improvement.

\section{The functional markers for the future}

It is necessary to use gene-specific markers for selection high GPC in wheat breeding. In this study, two complementary pairs of functional markers were developed to distinguish the two haplotypes of TaAAP6-3B. Lines carrying TaAAP6-3B-I exhibited significantly higher GPC than those carrying TaAAP6-3B-II in both established natural and genetic populations (Fig. 5). As developments of other favored allele's functional markers, containing quality traits, agronomic traits and disease resistance, the two functional markers could be used in Molecular Module-Based Designer Breeding Systems for wheat.

\section{Conclusions}

In conclusion, TaAAP6-3B, the ortholog of OsAAP6, was cloned and analyzed. The expression patterns, haplotypes, cis-regulatory elements and locus polymorphism were conducted to underlie its effect to GPC. TaAAP6-3B-I, the favored haplotype of TaAAP6-3B, is associated with significantly higher GPC than that ofTaAAP6-3B-II. Meanwhile, this haplotype has undergone the strong selection pressure in artificial selection during wheat breeding. This study provides important genes and functional markers for wheat breeding in nutritional quality improvement.

\section{Methods}

\section{Plant material and growth condition}

A total of 115 bread wheat lines, collected from our lab (Pro Tao Wang of Chengdu Institute of Biology, Chinese Academy of Sciences) were used for this study, including 64 landraces and 51 modern cultivars. Wheat plants were grown in two locations, Shuangliu (SL) $\left(103.87^{\circ} \mathrm{E}\right.$, $\left.30.59^{\circ} \mathrm{N}\right)$ and Shifang (SF) $\left(104.01^{\circ} \mathrm{E}, 31.29^{\circ} \mathrm{N}\right)$, in Sichuan Province by three growing seasons, 2010-2011, 2014-2015 and 2015-2016. In addition, a segregation population contains $197 \mathrm{~F}_{2}$ was grown in 2016-2017 growing season at Shuangliu. The lines were sown in single row of $1.2 \mathrm{~m}$ long and $0.2 \mathrm{~m}$ apart with three replications per site. Fields were managed according to local agricultural practices.

\section{Measurement of GPC and TKW}

After harvested, three plants of each line per replication were selected at random for phenotype analysis. Fully filled grains were used for measuring content of grain protein (GPC) and determined twice for each line by a Kjeltec 2200 system (FOSS). And, three independent samples of 200 grains were converted to one thousand-kernel weight (TKW) by SC-E software (Hangzhou Wanshen Detection Technology Co., Ltd., Hangzhou, China).

\section{DNA and RNA extraction}

Genomic DNA from all plant materials was extracted from young leaves by the CTAB method. Total RNA was extracted from various plant tissues with an RNA extraction kit and TRIzol reagent (Invitrogen). Firststrand cDNA was synthesized by $2 \mathrm{mg}$ RNA and $200 \mathrm{U}$ M-MLV reverse transcriptase (Promega) in 20ul volume, and subsequent for quantitative real-time PCR (qRT-PCR).

\section{Primers and PCR amplification}

All primers used in this study were designed by Primer Premier 5.0 software, are listed in (Additional file 1: Table S2), and synthesized in Chengdu TSINGKE Biological Technology Co., Ltd. PCR was performed in total volumes of $20 \mathrm{uL}$, including $50 \mathrm{ng}$ DNA, $0.5 \mathrm{uL} 10 \mathrm{mM}$ forward and reverse primers, $2 \mathrm{uL}$ of $25 \mathrm{mM}$ dNTP, $2 \mathrm{uL}$ GC buffer and $0.45 \mathrm{uL}$ KOD Plus polymerase (TOYOBO, Shanghai, China). PCR were performed as follows: $94{ }^{\circ} \mathrm{C}$ for $4 \mathrm{~min}$; followed by 35 cycles of $94{ }^{\circ} \mathrm{C}$ for $35 \mathrm{~s}$, annealing for $35 \mathrm{~s}$ and $72{ }^{\circ} \mathrm{C}$ for extension ( $30 \mathrm{~s}-2 \mathrm{~min}$ ); and $72^{\circ}$ $\mathrm{C}$ for $10 \mathrm{~min}$ with a final extension. The annealing temperature and extension time depended on the primer sets and the length of PCR products. All PCR products were separated via gel-electrophoresis on a $1.5 \%$ agarosegel stained with ethidium bromide and visualized by UV light. To examine the accuracy of primers, PCR products were selected for DNA sequencing and analysis.

\section{Isolate TaAAP6-3A, $3 B$ and $3 D$ in wheat}

The homologous sequence of OsAAP6 in wheat was obtained via blast in NCBI. Chinese Spring was used to clone the sequence of TaAAP6. Ninepairs of Primers $A F 1, A F 2, A F 3, B F 1, B F 2, B F 3, D F 1, D F 2$, and $D F 3$ were designed to amplify the Genome-specific fragments according to the blast sequence, and full length sequence of TaAAP6-3A, TaAAP6-3B, and TaAAP6-3D gene was assembled with the three fragments performed by DNAMAN. The PCR products werecloned into the pEASYBlunt simple vector and transformed to DH5 $\alpha$ competent $E$. coli cells by the heat shock method (Transgen, Beijing, China, Product Code: CT101). Positive clones were selected for sequencing (TSINGKE, Chengdu, China). PCR and positive clones were repeated at least three times.

\section{Phylogenetic and Cis-acting elements analysis}

Phylogenetic trees were constructed based on the fulllength amino acid sequences of TaAAP6-3B, and other AAP6s in plants. Phylogenetic analysis was performed by MEGA7.0 software, and using multiple algorithms (neighbor joining, minimum evolution, maximum likelihood, Nei and Kumar 2000) and different distance model (P versus PC distance) and gap options (complete 
versus pairwise deletion). Bootstrap values were estimated based on 1000 replications.

The core elements of the promoters were identified using the TSSP program. Cis-acting regulatory elements of the promoter were predicted by PLACE analysis.

\section{TaAAP6-3B haplotypes and functional markers \\ development}

In order to test the genotype of TaAAP6-3B alleles, three pairs of Primers $B F 1, B F 2$, and $B F 3$ were used to clone the sequence in 115 lines (Fig. 2). SNPs were identified using DNA Star software. Two markers (BI, BII) were designed by haplotypes to distinguish cultivars and $\mathrm{F}_{2}$ populations, respectively. Genome-specific fragments were amplified by the corresponding primers and then separated by electrophoresis in agarose-gel. To examine the accuracy of functional markers, PCR products were selected for DNA sequencing and analysis.

\section{Expression analysis}

Quantitative real-time PCR was carried out in a total volume of $20 \mathrm{ul}$ containing $0.5 \mathrm{ul}$ of the reverse-transcribed product, $0.4 \mathrm{ul}$ of genes specific primers $q A, q B, q D$ (Additional file 1: Table S2), 0.4 ul Passive Reference DyeII, $10 \mathrm{ul}$ of $2 \times$ Trans Start Top Green qpcrSuperMix (Transgen, Beijing, China) on an ABI 7500 real-time PCR system (Applied Biosystems, USA), according to the manufacturer's instructions. A sequence about 170 bp was amplified as a reference gene to continue. Measurements were obtained by means of the relative quantification method [32]. All expression level data obtained by quantitative real-time PCR are based on at least four biological samples on which three replications of the technique were conducted.

\section{TaAAP6-3B locus polymorphism}

The size of $3 \mathrm{~B}$ chromosome contents $\sim 740 \mathrm{Mb}$, and 79 locus blocks [30], which means the average size of one block contains $\sim 10 \mathrm{Mb}$. As a result, each $\sim 5 \mathrm{Mb}$ upstream and downstream the TaAAP6-3B was selected from the wheat genome to analysis the Locus polymorphism. 40 SNPs were found in this region from the high-density wheat $90 \mathrm{~K}$ Illumina iSelect array in 3B chromosome. These SNPs were used for genotyping in 115 wheat lines at the Compass Biotechnology Co., Ltd.

\section{Statistical analyses}

To determine phenotypic differences between genotypes, all data obtained were subjected to ANOVA, and the mean difference was compared by the LSD test at $95 \%$ levels of probability. Sequences were analyzed by DNAMAN software. In all figures, the spread of values is shown as error bars representing standard errors of the means.

\section{Additional files}

Additional file 1: Table S2. Primers for cloning and functional analysis of TaAAP6-3B. (DOCX $15 \mathrm{~kb}$ )

Additional file 2: Figure S1. Sequence alignment of OSAAP6 and three TaAAP6 homoeologues. (PPTX $795 \mathrm{~kb}$ )

Additional file 3: Table S1.TaAAP6-3B genotypes and Locus polymorphism in 115 wheat lines. (DOCX $31 \mathrm{~kb}$ )

Additional file 4: Table S4. Putative cis-regulatory elements detected in TaAAP6-3B-I. (DOCX $15 \mathrm{~kb}$ )

Additional file 5: Figure S2. Comparative expression pattern of TaAAP6-3B. (PPTX 77 kb)

Additional file 6: Table S3. Comparisons the GPC and TKW between TaAAP6-3B-I and TaAAP6-3B-/l about 115 lines in five environments. (DOCX $18 \mathrm{~kb}$ )

Additional file 7: Table S5. Contents of protein grain from genotypic classes among 197 individuals from a $F_{2}$ population. (DOCX $15 \mathrm{~kb}$ )

\section{Abbreviations}

ANOVA: Analysis of variance; CREs: Cis-regulatory elements; DAF: Days after flowering; GPC: Content of grain protein; NCBI: National Center for Biotechnology Information; QTL: Quantitative trait loci; TKW: Thousand kernel weight

\section{Acknowledgements}

We thank Wuyun Yang for providing some of the wheat landraces seeds.

\section{Funding}

This work was supported by grants from the National Key Project of Transgenic Biologic Varieties Breeding of China (Grant No. 2016ZX08009003004), the National Basic Research Program of China ( 973

Program, 2014CB138104-2), and the National Key Research and

Development Program of China (Grant No.2016YFD0102000).

Availability of data and materials

All the data supporting our findings is contained within the manuscript.

\section{Authors' contributions}

$X-F J$ carried out most of the experiments and wrote the manuscript, T-W coordinated the project, conceived and designed experiments. B-F conducted the bioinformatics work, generated and analyzed data, and edits the manuscript. Z-BX and F-XL collected the samples; J-L, Q-L and P-Z performed the laboratory work. All authors read and approved the final manuscript.

\section{Ethics approval}

The source of our grain seeds were from the Pro Tao Wang of Chengdu Institute of Biology, Chinese Academy of Sciences. The experimental research on plants, including collection of plant material, was complied with institutional, national, or international guidelines. And field studies were conducted in accordance with local legislation.

\section{Competing interests}

The authors declare they have no competing interests.

\section{Publisher's Note}

Springer Nature remains neutral with regard to jurisdictional claims in published maps and institutional affiliations.

\section{Author details}

${ }^{1}$ Chengdu Institute of Biology, Chinese Academy of Sciences, Chengdu, China. ${ }^{2}$ University of Chinese Academy of Sciences, NO.19 Yuquan Road, Beijing, China. 
Received: 16 October 2017 Accepted: 3 April 2018

Published online: 23 April 2018

\section{References}

1. Uauy C, Distelfeld A, Fahima T, Blechl A, Dubcovsky J. A NAC gene regulating senescence improves grain protein, zinc, and iron content in wheat. Science. 2006;314(5803):1298-301.

2. Balyan HS, Gupta PK, Kumar S, Dhariwal R, Jaiswal V, Tyagi S, Agarwal P, Gahlaut V, Kumari S. Genetic improvement of grain protein content and other health-related constituents of wheat grain. Plant Breed. 2013; 132(5):446-57.

3. Peng B, Kong H, Li Y, Wang L, Zhong M, Sun L, Gao G, Zhang Q, Luo L, Wang G. OsAAP6 functions as an important regulator of grain protein content and nutritional quality in rice. Nat Commun. 2014;5(1):4847.

4. Mayer KFX, Rogers J, Doležel J, Pozniak C, Eversole K, Feuillet C, Gill B, Friebe B, Lukaszewski AJ, Sourdille P. A chromosome-based draft sequence of the hexaploid bread wheat (Triticum aestivum) genome. Science. 2014; 345(6194):1251788.

5. Lin Q, Hao C, Jian H, Wang Y, Tian L, Wang L, Ma Z, Zhang X. Homologous haplotypes, expression, genetic effects and geographic distribution of the wheat yield gene TaGW2. BMC Plant Biol. 2014;14(1):107.

6. Ma L, Li T, Hao C, Wang Y, Chen X, Zhang X. TaGS5-3A, a grain size gene selected during wheat improvement for larger kernel and yield. Plant Biotechnol J. 2016;14(5):1269-80.

7. Jiang Y, Jiang Q, Hao C, Hou J, Wang L, Zhang H, Zhang S, Chen X, Zhang $X$. A yield-associated gene TaCWl, in wheat: its function, selection and evolution in global breeding revealed by haplotype analysis. Theor Appl Genet. 2015;128(1):131.

8. Deol KK, Mukherjee S, Gao F, Brûlébabel A, Stasolla C, Ayele BT. Identification and characterization of the three homeologues of a new sucrose transporter in hexaploid wheat (Triticum aestivum L.). BMC Plant Biol. 2013;13(1):1-15

9. Joppa LR. Mapping gene(s) for grain protein in Tetraploid wheat (Triticum turgidum L.) using a population of recombinant inbred chromosome lines. Crop Sci. 1997;37(5):1586-9.

10. Prasad M, Kumar A, Balyan H, PC EK, Singh H, Dhaliwal H, Roy J, Gupta P, Varshney R. A microsatellite marker associated with a QTL for grain protein content on chromosome arm 2DL of bread wheat. Theor Appl Genet. 1999; 99(2):341-5.

11. Perretant MR, Cadalen T, Charmet G, Sourdille P, Nicolas P, Boeuf C, Tixier MH, Branlard G, Bernard S. QTL analysis of bread-making quality in wheat using a doubled haploid population. Theor Appl Genet. 2000;100(8):1167-75.

12. Zanetti S, Winzeler M, Feuillet C, Keller B, Messmer M. Genetic analysis of bread_aking quality in wheat and spelt. Plant Breed. 2010;120(1):13-9.

13. Wang S, Wong D, Forrest K, Allen A, Chao S, Huang BE, Maccaferri M, Salvi S, Milner SG, Cattivelli L. Characterization of polyploid wheat genomic diversity using a high-density 90000 single nucleotide polymorphism array. Plant Biotechnol J. 2014;12(6):787.

14. Tegeder M. Transporters for amino acids in plant cells: some functions and many unknowns. Curr Opin Plant Biol. 2012;15(3):315.

15. Wan Y, King R, Mitchell RAC, Hassanipak K, Hawkesford MJ. Spatiotemporal expression patterns of wheat amino acid transporters reveal their putative roles in nitrogen transport and responses to abiotic stress. Sci Rep. 2017;7(1):5461.

16. Kai ZX, Liang ZJ, Nian FC, Shan GW, Xin PY. Differences of protein and its component accumulation in wheat for different end uses. Acta Agron Sin. 2005;31(3):342-7.

17. Doebley J, Lukens L. Transcriptional regulators and the evolution of plant form. Plant Cell. 1998;10(7):1075.

18. Ulmasov T, Hagen G, Guilfoyle TJ. Activation and repression of transcription by auxin-response factors. Proc Natl Acad Sci U S A. 1999;96(10):5844.

19. Okushima Y, Overvoorde PJ, Arima K, Alonso JM, Chan A, Chang C, Ecker JR, Hughes B, Lui A, Nguyen D. Functional genomic analysis of the AUXIN RESPONSE FACTOR gene family members in Arabidopsis thaliana: unique and overlapping functions of ARF7 and ARF19. Plant Cell. 2005:17(2):444-63.

20. Allen RD, Bernier F, Lessard PA, Beachy RN. Nuclear factors interact with a soybean beta-conglycinin enhancer. Plant Cell. 1989;1(6):623.

21. Salamini F, Ozkan H, Brandolini A, Schäferpregl R, Martin W. Genetics and geography of wild cereal domestication in the near east. Nat Rev Genet. 2002;3(6):429-41.
22. Zhang LZ, Tan QM, Lee R, Trethewy A, Lee YH, Tegeder M. Altered xylemphloem transfer of amino acids affects metabolism and leads to increased seed yield and oil content in Arabidopsis. Plant Cell. 2010;22(11):3603.

23. Zhao H, Ma H, Yu L, Wang X, Zhao J. Genome-wide survey and expression analysis of amino acid transporter gene family in Rice ( Oryza sativa L.). PLoS One. 2012;7(11):e49210.

24. Swinnen $G$, Goossens A, Pauwels L. Lessons from domestication: targeting Cis-regulatory elements for crop improvement. Trends Plant Sci. 2016;21(6):506-15.

25. Lev-Yadun S, Gopher A, Abbo S. The cradle of agriculture. Science. 2000; 288(5471):1602-3.

26. Reif JC, Zhang P, Dreisigacker S, Warburton ML, Van GM, Hoisington D, Bohn $M$, Melchinger AE. Wheat genetic diversity trends during domestication and breeding. Theor Appl Genet. 2005;110(5):859.

27. Blanco A, Mangini G, Giancaspro A, Giove S, Colasuonno P, Simeone R, Signorile A, Vita PD, Mastrangelo AM, Cattivelli L. Relationships between grain protein content and grain yield components through quantitative trait locus analyses in a recombinant inbred line population derived from two elite durum wheat cultivars. Mol Breed. 2012;30(1):79-92.

28. Groos C, Robert N, Bervas E, Charmet $G$. Genetic analysis of grain proteincontent, grain yield and thousand-kernel weight in bread wheat. Theor Appl Genet. 2003:106(6):1032.

29. Rajaram IJRPKSS: CIMMYT's genetic progress in wheat grain quality under four nitrogen rates. Crop Sci 1997, 38(2):7.

30. Gardner KA, Wittern LM, Mackay IJ. A highly recombined, high-density, eight-founder wheat MAGIC map reveals extensive segregation distortion and genomic locations of introgression segments. Plant Biotechnol J. 2016;14(6):1406-17.

31. Marone D, Laidò G, Gadaleta A, Colasuonno P, Ficco D, Giancaspro A, Giove S, Panio G, Russo M, Vita P. A high-density consensus map of A and B wheat genomes. Theor Appl Genet. 2012;125(8):1619.

32. Livak KJ, Schmittgen TD. Analysis of relative gene expression data using real-time quantitative PCR and the 2(-Delta Delta $C(T))$ method. Methods. 2012;25(4):402-8.

\section{Ready to submit your research? Choose BMC and benefit from:}

- fast, convenient online submission

- thorough peer review by experienced researchers in your field

- rapid publication on acceptance

- support for research data, including large and complex data types

- gold Open Access which fosters wider collaboration and increased citations

- maximum visibility for your research: over $100 \mathrm{M}$ website views per year

At BMC, research is always in progress.

Learn more biomedcentral.com/submissions 